\title{
Proton-pump inhibitor overuse: a cautionary tale in misguided benefit
}

\author{
Karthik Nath ${ }^{1}$, Reza Reyaldeen ${ }^{1,2}$, Dharmenaan Palamuthusingam ${ }^{1,3}$ \\ ${ }^{1}$ Faculty of Medicine, University of Queensland \\ ${ }^{2}$ Department ofCardiology, Princess Alexandra Hospital \\ ${ }^{3}$ Department ofNephrology, Logan Hospital, Brisbane, Queensland, Australia
}

We congratulate Leitinger et al.1 and co-authors for their comprehensive review on the use of proton-pump inhibitors (PPI) in patients with malignant haematological conditions. The authors highlight the high prophylactic and often extended use of PPI in patients receiving treatment for multiple myeloma, lymphoma and recipients of autologous haemopoietic stem cell transplantation. In those with multiple myeloma or lymphoma there was no strong indication for the initiation of PPI in more than half the patients studied. Furthermore, therapy was typically continued for a protracted duration, sometimes indefinitely despite no indication for therapy. Studies involving the general population from the United States and the United Kingdom have also reported up to $70 \%$ of PPI prescription without indication.2, 3 The authors have also highlighted the paucity of robust evidence on the indications for prophylaxis in those patients receiving glucocorticoid therapy. Their national survey demonstrated high variability in clinical practice and an overall lack of institutional protocols for the use of PPI therapy in this setting.

PPI therapy has been widely regarded as safe and well tolerated, and it seems this belief perpetuates its use beyond the desired indication. The work by Leitinger et al.1 increases the awareness of the potential toxicities of PPI therapy. However, an important and increasingly recognised association of kidney disease in the setting of PPI overuse needs to be highlighted. Several large multinational observational studies have consistently demonstrated an association between PPI use and development of chronic kidney disease (CKD), progression of CKD and development of end-stage kidney disease, even without intervening acute kidney injury.3, 4 Studies have also suggested a graded relationship between kidney injury and duration of drug exposure.4 However, the mechanism of injury remains to be elucidated, but appears to be driven by an interstitial pathology rather than glomerular disease itself.4 These findings reiterate the importance of judicious use of PPI in an already vulnerable patient population who remain at risk of kidney disease from the malignancy and chemotherapy itself.5 With an ever-increasing repertoire of novel cancer therapies demonstrating improved survival outcomes, there needs to be a greater focus on survivorship and long-term treatment toxicities. 
We fully support Leitinger et al.1 in their call for increased pharmacovigilance when prescribing PPI, not only in patients being treated for haematological malignancies, but also other medical patients as well. These concerns should not deter clinicians from prescribing PPI when indicated, but should reinforce the need for a measured approach in prescription and de-escalation.

In contrast to disease-modifying treatments, supportive care in malignant haematology is often not reinforced by a strong evidence-based approach. We hope that studies such as this, encourage institutions and national bodies to develop robust, evidence-based guidelines on the safe prescription of supportive therapies in cancer.

\section{References}

1. Leitinger $E$, Hui L, Grigg A. Is there a role for proton pump inhibitor prophylaxis in haematology patients? Intern Med J 2019; 49: 694- 701.

2. Forgacs I, Loganayagam A. Overprescribing proton pump inhibitors. BMJ 2008; 336: 2-3.

3. Lazarus B, Chen Y, Wilson FP, Sang Y, Chang AR, Coresh J et al. Proton pump inhibitor use and the risk of chronic kidney disease. JAMA Intern Med 2016; 176: 238- 46.

4. Xie Y, Bowe B, Li T, Xian H, Balasubramanian S, Al-Aly Z. Proton pump inhibitors and risk of incident CKD and progression to ESRD. J Am Soc Nephrol 2016; 27: 3153-63.

5. Ganguli A, Sawinski D, Berns JS. Kidney diseases associated with haematological cancers. Nat Rev Nephrol 2015; 11: 478- 90. 